\title{
"New" metastases are associated with a poorer prognosis than growth of pre- existing metastases in patients with metastatic breast cancer treated with chemotherapy
}

Christopher Twelves ${ }^{1,12^{*}}$, Javier Cortes ${ }^{2,3}$, Peter A. Kaufman ${ }^{4}$, Louise Yelle ${ }^{5}$, Ahmad Awada ${ }^{6}$, Terri A. Binder ${ }^{7}$, Martin Olivo ${ }^{8}$, James Song ${ }^{9}$, Joyce A. O'Shaughnessy ${ }^{10}$, Maria Jove ${ }^{1}$ and Edith A. Perez ${ }^{11}$

\begin{abstract}
Introduction: Progression-free survival (PFS) and overall survival (OS) endpoints often only weakly correlate. This analysis investigates how different progression events impact on OS, using data from two phase 3 studies with eribulin in women with advanced/metastatic breast cancer (MBC).

Methods: In Study 301, 1102 women with $\leq 2$ prior chemotherapies for advanced/MBC were randomized to eribulin mesylate (1.4 mg/m $\mathrm{m}^{2}$ on days 1 and 8 every 21 days) or capecitabine $\left(1.25 \mathrm{~g} / \mathrm{m}^{2}\right.$ twice daily on days $1-14$ every 21 days). Study 305/EMBRACE enrolled 762 patients following two to five prior chemotherapies for advanced/MBC, randomized to eribulin (as above) or treatment of physician's choice. We analyzed OS and PFS post hoc for patients whose disease progressed due to development of "new" metastases, growth of pre-existing lesions, and patients with no reported disease progression.
\end{abstract}

Results: In both clinical studies, development of new metastases was associated with an increased risk of death $(p<0.0001)$. The time to development of new metastasis or death was significantly longer with eribulin than the comparator in Study 305 ( $p=0.0017)$, but not in Study $301(p=0.46)$. Significantly longer OS was observed in the eribulin compared with the comparator arm for the new metastases subgroup in Study $301(p=0.008)$, but not in Study 305 ( $p=0.16)$, compared with other progression subgroups.

Conclusions: Patients with MBC progressing with new metastases have a worse prognosis than those whose disease progresses due to growth of existing lesions or patients with no reported disease progression. These findings have potentially important implications for the interpretation of clinical study data and clinical practice.

Trial registration: ClinicalTrials.gov registration IDs: Study 301: NCT00337103; Study 305: NCT00388726.

\footnotetext{
* Correspondence: C.J.Twelves@leeds.ac.uk

'Section of Oncology and Clinical Research, Leeds Institute of Cancer and

Pathology, University of Leeds and Leeds Teaching Hospitals Trust, Leeds, UK

${ }^{12}$ St James' Institute of Oncology, St James' University Hospital, University of

Leeds, Bexley Wing, Level 4, Beckett Street, Leeds, UK

Full list of author information is available at the end of the article
}

C Biomed Central

(C) 2015 Twelves et al. Open Access This article is distributed under the terms of the Creative Commons Attribution 4.0 International License (http://creativecommons.org/licenses/by/4.0/), which permits unrestricted use, distribution, and reproduction in any medium, provided you give appropriate credit to the original author(s) and the source, provide a link to the Creative Commons license, and indicate if changes were made. The Creative Commons Public Domain Dedication waiver (http://creativecommons.org/publicdomain/zero/1.0/) applies to the data made available in this article, unless otherwise stated. 


\section{Introduction}

Overall survival (OS) has been regarded as the goldstandard endpoint in phase 3 cancer studies since the 1980s when it replaced objective response rate (ORR) as the principal endpoint for studies supporting anticancer drug approval $[1,2]$. OS benefits can, however, be difficult to assess because they may require a large patient population and a prolonged follow-up period to demonstrate a statistically significant difference [3, 4]. Additionally, in clinical studies evaluating therapy earlier in the course of metastatic disease, OS may be strongly influenced by subsequent lines of therapy $[5,6]$. Many phase 3 oncology studies, including those in metastatic breast cancer (MBC), have therefore used alternative primary endpoints (also known as intermediate endpoints) such as progression-free survival (PFS) [3]. Use of PFS markedly reduces the number of patients necessary to establish a statistically significant benefit, particularly when the time between the progression event and death may be relatively long [4] or when there is extensive crossover [7].

Despite widespread use in clinical trials, many intermediate endpoints are poorly defined or have varying definitions in the literature, making it difficult to consistently interpret treatment effects and reliably perform cross-trial comparisons. The heterogeneity of definitions for endpoints has triggered the recent DATECAN (Definition for the Assessment of Time-to-Event Endpoints in CANcer trials) initiative, aimed at standardizing consensus definitions of endpoints for multiple cancer sites, including breast cancer [8].

Meta-analyses of intermediate endpoints, such as PFS, have suggested that these are often not reliable surrogate endpoints for OS in MBC [3]. A recent United States Food and Drug Administration evaluation of 12 clinical studies submitted in support of approvals for treatments for MBC suggested that PFS and OS are only weakly correlated $\left(R^{2}=0.079\right)$ [9]. This poor correlation may be due, at least in part, to crossover and variations in poststudy anticancer therapies [7].

It can, therefore, be difficult to interpret the true clinical impact of a new agent that improves PFS but not OS, particularly when other treatments with established OS benefits are available [10]. Thus, there is an ongoing debate as to whether benefits in PFS, in the absence of increases in OS, are clinically relevant [11]. By contrast, while less common historically, increases in OS without improvements in PFS have been reported in phase 2 and 3 studies of immunotherapies [12, 13] and with an endothelin receptor antagonist $[14,15]$ in patients with castration-resistant metastatic prostate cancer, as well as in a phase 3 study comparing antiepidermal growth factor receptor and antivascular endothelial growth factor therapies for metastatic colorectal cancer [16]. Further, it is increasingly questioned whether PFS, as conventionally defined, is a meaningful endpoint in clinical studies of immunotherapies, where a tumor "flare-characterized by active inflammation, enhancement and even potentially increased tumor mass - may be seen before a conventional response has had time to develop [17-19].

Improvements in OS, but not PFS, have been observed in only a few clinical studies of cytotoxic chemotherapy [20-22]. In two recent, large-scale, randomized, phase 3 clinical studies of the nontaxane microtubule dynamics inhibitor eribulin, patients in the eribulin arm appeared to benefit more in terms of OS than PFS [23, 24]. One of these studies (Study 305/Eisai Metastatic Breast Cancer Study Assessing Physician's Choice Versus E7389 [EMBRACE]) [23] demonstrated a significant increase in the primary endpoint of OS with eribulin compared with treatment of physician's choice (TPC) in women with $\mathrm{MBC}$ who had received between two to five previous chemotherapy regimens, including $\geq 2$ for advanced disease (median 13.1 vs. 10.6 months; hazard ratio [HR] $0.81 ; 95 \%$ confidence interval $[\mathrm{CI}] 0.66,0.99 ; p=0.041$ ). There was a small but significant difference in favor of eribulin in PFS as assessed by investigator (HR 0.76; $95 \%$ CI $0.64,0.90 ; p=0.002)$, but not independent review (HR 0.87; $95 \%$ CI 0.71, 1.05; $p=0.137$ ), likely due to fewer patients being censored with investigator review, resulting in more progression events, compared with independent review. The median PFS values were, however, similar for the independent and investigator reviews [23].

A separate clinical study (Study 301) compared eribulin with capecitabine in patients with $\mathrm{MBC}$ who had received up to three prior chemotherapy regimens, including $\leq 2$ for advanced or metastatic disease [24]. This study showed a numerical difference favoring improved OS with eribulin compared with capecitabine; although this difference was not statistically significant (median 15.9 vs. 14.5 months; HR 0.88; $95 \%$ CI 0.77, 1.00; $p=0.056$ ). PFS was almost identical in both treatment arms (median 4.1 vs. 4.2 months; HR 1.08; $95 \%$ CI 0.93, 1.25; $p=0.30$ ) [24]. Sensitivity analyses adjusting for a crossover effect and postprogression anticancer treatments were consistent with the primary analysis [25], suggesting that neither of these fully explain the apparent discordance between OS and PFS.

In response to the challenges of interpreting PFS or disease-free survival (DFS) data, the European Medicines Agency (EMA) recently published guidelines for consideration when using these endpoints in clinical studies [26]. PFS is a composite endpoint, including the appearance of "new" lesions (i.e., newly detected metastases, in a new or pre-existing metastatic site), progression of existing metastases (i.e., those present at baseline), mixed responses with some lesions increasing in size but others decreasing in size, and death. The EMA guidelines recommend the use of 
separate analyses that disassemble PFS into specific progression events. Accordingly, we have performed post hoc analyses of data from Study 301 and Study 305/EMBRACE to assess whether the study drugs had different effects on OS according to the type of progression event, and to explore the apparent discordance between OS and PFS.

Our first aim was to investigate whether the type of progression event was predictive for OS in Study 301 and Study 305/EMBRACE. In addition, we investigated whether a modified PFS, "new-metastasis-free survival," defined as the time from randomization to progression due to a "new" metastasis or death, renders better concordance with OS between the eribulin and control (TPC or capecitabine) arms.

\section{Methods}

The clinical study design and efficacy and safety results of Study 301 [24] and Study 305/EMBRACE [23] have been reported elsewhere. Key eligibility criteria and treatment details are summarized briefly here. All patients gave written, informed consent. Approval was obtained from independent ethics committees and regulatory authorities in participating countries (Appendix I). The studies were conducted in accordance with the World Medical Association (WMA) Declaration of Helsinki (WMA General Assembly, Tokyo, 2004) guidelines of the Committee for Proprietary Medicinal Products/International Conference for Harmonisation/Good Clinical Practice (CPMP/ICH/135/95), and local ethical and legal requirements.

\section{Clinical study designs}

\section{Study 301 [24]}

Patients who had received up to three prior chemotherapy regimens, including up to two regimens for advanced and/ or metastatic disease, were eligible; prior therapy must have included an anthracycline and a taxane. Eligible patients were randomized $1: 1$ to $1.4 \mathrm{mg} / \mathrm{m}^{2}$ eribulin mesylate intravenously (equivalent to $1.23 \mathrm{mg} / \mathrm{m}^{2}$ eribulin [as free base]) on days 1 and 8 of a 21-day cycle, or capecitabine $1.25 \mathrm{~g} / \mathrm{m}^{2}$ orally twice daily on days $1-14$ of a 21 day cycle. Patients were stratified by geographic region and human epidermal growth factor receptor 2 status.

\section{Study 305/EMBRACE [23]}

Patients were required to have received two to five previous chemotherapy regimens, including an anthracycline and a taxane, with at least two regimens for locally recurrent breast cancer or MBC. Eligible patients were randomized 2:1 to eribulin (using the same dose and schedule as in Study 301) or TPC, defined as: any singleagent chemotherapy, hormonal, or biological treatment approved for the treatment of cancer; radiotherapy; or symptomatic treatment alone. Patients were stratified by geographical region, previous capecitabine use, and human epidermal growth factor receptor 2 status.

\section{Post hoc analysis of PFS and OS}

Progression events (determined using Response Evaluation Criteria In Solid Tumors [RECIST] version 1.0) [27] from both clinical studies were categorized as: detection of a new lesion/metastasis (defined as a lesion not identified at baseline); increase in the sum of the longest diameters of preexisting target lesions (i.e., those present at baseline) or an increase in the size of at least one nontarget lesion reported at baseline; and patients with no reported disease progression that includes death, clinical deterioration, and patients who were censored. If progressive disease was detected objectively (radiologically or by objective clinical measurement of skin lesions), patients in whom a new lesion was detected were classified as such, even if there was also progression of existing lesions. Based on the above classification, new-metastasis-free survival, defined as the time from randomization to death or progression due to a new metastasis (whichever occurred earlier), was used to investigate the treatment effect in the progression type of special interest, and to better understand the potential relationship between disease progression and OS. The time to new metastasis in vital organs (central nervous system [CNS], lungs, or liver) was also calculated because we hypothesized that such metastases may have a particular impact on outcomes.

Independent review of disease progression in the intent-to-treat (ITT) population was used for the primary analyses. Separate analyses were conducted with investigator review (data not shown); however, results by independent review only are presented here because they are considered to be more reliable. Moreover, because PFS from independent reviews showed discordance with OS in both studies, it is further appropriate to explore the reasons of such discordance using the independent review data.

\section{Statistical analyses}

The correlation between progression events and OS was investigated by Cox regression, incorporating the development of a new lesion/metastasis as a time-dependent covariate. OS in the different treatment groups was compared by PFS status using Cox regression and stratified log-rank tests. In the analysis of new-metastasisfree survival, the cumulative incidence competing risk (CICR) method [28] was used to estimate the cumulative probabilities of disease progression due to the development of a new lesion or death, in which progression due to existing lesion was considered as a competing risk. The CICR method (unlike the Kaplan-Meier estimator) does not require any assumption about independence of the competing risks. The independence assumption required by logrank test implies that at each time point the hazard of 
developing new metastases or death is the same for patients who were in follow-up and had no event, and for patients who died due to growth of a pre-existing lesion by that time, which cannot be verified in our studies. The unstratified Gray's test [29] was used to compare the cumulative incidence functions between the two treatment arms in the competing risks setting. Because death or progression due to an existing lesion or nonvital new lesion was censored by definition in the calculation of time to new metastasis in vital organs (i.e., competing risks are not considered), Kaplan-Meier estimator and log-rank tests were used to estimate the treatment difference.

\section{Results}

Study 301 comprised 1102 patients in the ITT population, with 554 in the eribulin arm and 548 in the capecitabine arm. Study 305/EMBRACE comprised 762 patients in the ITT population, with 508 in the eribulin arm and 254 in the TPC arm. Consolidated Standards of Reporting Trials (CONSORT) diagrams for both clinical studies have been published previously [23, 24]. The results described below are based on tumor assessment by independent review; however, similar results were obtained with investigator review (data not shown), unless stated otherwise.

\section{Study 301}

\section{Prognostic effects of type of progression}

Of the 1102 patients in the ITT population, 1101 had baseline scans, and $420(38.1 \%)$ patients had disease progression due to the development of new metastases. Patients who were deemed to have tumor progression due to new metastases had an increased risk of death compared with all other patients $(n=682$; median OS 13.0 months vs. 17.1 months, respectively), regardless of whether the data were stratified by treatment group or not (HR 1.98 and 1.96; $p<0.0001$; Tables 1 and 2).

Similar findings were observed when median OS was compared between patients whose disease progressed due to new metastases and those whose disease progressed specifically due to an increase in the size of pre-existing lesions ( $n=420$ vs. 243 , respectively; 13.0 months vs. 17.1 months, respectively), regardless of stratification (HR 1.81 and 1.80; $p<0.0001$; Tables 1 and 2). Median OS was also shorter for patients who developed new metastases than for those patients with no reported disease progression (which includes death, clinical deterioration and censoring; [ $n=439$ ], 13.0 months vs. 16.9 months, respectively) irrespective of stratification (HR 2.20 and 2.16, $p<0.0001$; Tables 1 and 2).

\section{Comparison of eribulin versus capecitabine according to progression event}

Disease progression due to new metastases occurred in 216 patients $(39.0 \%)$ in the eribulin arm compared with 204 patients $(37.2 \%)$ in the capecitabine arm. In the eribulin arm, 131 patients (23.7\%) progressed due to an increase in the size of pre-existing lesions, compared with 112 (20.4\%) in the capecitabine arm. Two hundred seven (37.4\%) patients in the eribulin arm, which represented 148 (26.7\%) deaths, and 232 (42.3\%) patients in the capecitabine arm, which represented 179 (32.7\%) deaths, were associated with no reported disease progression.

Median OS for patients whose disease progressed due to new metastases was significantly longer with eribulin than with capecitabine ( 14.6 vs. 11.3 months; HR $0.75 ; p=0.008$; Table 2). For those whose disease progressed due to an increase in the size of pre-existing lesions, median OS was 18.0 versus 16.7 months (HR 0.92; $p=0.57$; Table 2). In patients with no reported disease progression, median OS was 17.6 versus 16.8 months (HR $0.91 ; p=0.44$ ) for eribulin and capecitabine, respectively.

Cumulative probabilities of new metastasis or death were similar in both arms (Gray's test $p=0.46$; Fig. 1a). Sensitivity analysis of new-metastasis-free survival using log-rank test showed similar results (median 5.8 vs. 5.8 months; HR 1.00; $p=0.99$; Fig. 2a). For patients whose disease progressed due to new metastases, there were no notable differences in the incidence of new metastases in different organs (Table 3). In addition, the time to new metastases in patients whose disease progressed due to new metastases in vital organs (CNS, lungs, or liver) was also not significantly different between the two treatment arms (HR 0.81; $p=0.12$; Fig. 3a).

\section{Comparison of eribulin versus capecitabine according to treatment duration}

No significant differences in treatment duration were observed between the patients treated with eribulin versus capecitabine irrespective of the type of progression event subgroup ( $p=\mathrm{ns}$ for all; data not shown).

\section{Study 305/EMBRACE}

\section{Prognostic effect of type of progression}

The ITT population comprised 762 patients, of whom 760 had baseline scans and 207 (27.2 \%) had disease progression due to new metastases. Similar to Study 301, patients with tumor progression due to new metastases were at an increased risk of death compared with all other patients $(n=555)$, whether stratified by treatment group or not (median OS 11.1 vs. 12.5 months, respectively; HR 2.25 and 2.27, respectively; $p<0.0001$; Tables 1 and 4 ).

The same differences in median OS were also observed for disease progression due to a new metastases compared with progression specifically due to an increase in the size of pre-existing lesions $(n=262$; median OS 11.1 vs. 12.2 months, respectively), whether stratified by treatment group or not (HR 2.09 and 2.12, respectively; $p<0.0001$; Tables 1 and 4). Patients in the new metastases subgroup also had an increased risk of death compared with the 
Table 1 Disease progression due to new metastasis as a risk factor of overall survival (intent-to-treat population)

\begin{tabular}{|c|c|c|c|c|}
\hline & \multicolumn{2}{|l|}{ Study 301} & \multicolumn{2}{|l|}{ Study 305/EMBRACE } \\
\hline & Stratified by treatment group & \multirow[t]{2}{*}{ Not stratified } & Stratified by treatment group & \multirow[t]{2}{*}{ Not stratified } \\
\hline \multicolumn{3}{|c|}{ Compared with all other ${ }^{a}$ subjects } & & \\
\hline Patients, n & 1102 & & 762 & \\
\hline HR $(95 \% \mathrm{Cl})$ & $1.98(1.71,2.29)$ & $1.96(1.70,2.27)$ & $2.25(1.79,2.83)$ & $2.27(1.80,2.85)$ \\
\hline Wald $p$ value & $<0.0001$ & $<0.0001$ & $<0.0001$ & $<0.0001$ \\
\hline \multicolumn{5}{|c|}{ Compared with those whose disease progressed due to an increase in the size of pre-existing lesion(s) } \\
\hline Patients, n & 663 & & 469 & \\
\hline HR $(95 \% \mathrm{Cl})$ & $1.81(1.54,2.14)$ & $1.80(1.53,2.13)$ & $2.09(1.63,2.68)$ & $2.12(1.65,2.72)$ \\
\hline Wald $p$ value & $<0.0001$ & $<0.0001$ & $<0.0001$ & $<0.0001$ \\
\hline \multicolumn{5}{|c|}{ Compared with those with no reported disease progression ${ }^{\text {b }}$} \\
\hline Patients, n & 859 & & 500 & \\
\hline HR $(95 \% \mathrm{Cl})$ & $2.20(1.88,2.57)$ & $2.16(1.85,2.53)$ & $2.61(2.03,3.37)$ & $2.60(2.02,3.34)$ \\
\hline Wald $p$ value & $<0.0001$ & $<0.0001$ & $<0.0001$ & $<0.0001$ \\
\hline
\end{tabular}

Tumor progression due to new metastasis is fitted as a time-dependent variable in the Cox regression model

Cl confidence interval, EMBRACE Eisai Metastatic Breast Cancer Study Assessing Physician's Choice Versus E7389, HR hazard ratio

a.e., progression due to growth of pre-existing lesion(s), or those with no reported disease progression (including death, clinical deterioration or censoring)

bPatients with no reported disease progression included death, clinical deterioration, or censoring

patients with no reported disease progression (that includes death, clinical deterioration, and censoring), ( $n=293]$; 11.1 vs. 13.0 months, respectively) irrespective of stratification (HR 2.61 and 2.60; $p<0.0001$; Tables 1 and 4).

\section{Comparison of eribulin versus TPC according to progression event}

Disease progression due to new metastases occurred in 139 patients $(27.4 \%)$ in the eribulin arm and 68 patients (26.8\%) in the TPC arm; an increase in the size of preexisting lesions occurred in 189 (37.2 \%) and 73 (28.73\%) patients receiving eribulin and TPC, respectively. In the eribulin arm, there were 180 (35.4\%) patients with no reported disease progression, compared with 113 (44.5\%) patients in the TPC arm which comprised 85 (16.7\%) and 61 (24.0\%) deaths in the eribulin and TPC arms, respectively. The proportion of patients whose disease progressed due to new metastases was lower in Study 305/EMBRACE than in Study 301 (27.2 \% vs. $38.1 \%$ ).

Median OS in the eribulin arm was not statistically different from that in the TPC arm for patients whose disease progressed due to new metastases (12.3 vs. 8.4 months; HR 0.76 ; $p=0.16$; Table 4), for those whose disease progressed due to an increase in the size of pre-existing lesions (13.0 vs. 11.1 months; HR $0.74 ; p=0.14$; Table 4 ) or those with no reported disease progression (15.8 vs. 10.7 months; HR $0.77 ; p=0.16$; Table 4). Although, in the investigator review analysis, the difference in median OS with eribulin compared with the TPC arm in patients with no reported disease progression reached statistical significance (16.1 vs. 9.9 months; HR 0.66; $p=0.03$ ).

New-metastasis-free survival was longer in the eribulin arm compared with the TPC arm, as indicated by the lower cumulative incidence rate curve of the eribulin arm (Fig. 1b). The differences in cumulative functions between the two arms were statistically significant (Gray's test, $p=$

Table 2 Overall survival for different patient groups in Study 301

\begin{tabular}{|c|c|c|c|c|c|c|c|c|c|}
\hline & \multicolumn{3}{|c|}{ Progression due to new metastases } & \multicolumn{3}{|c|}{ Progression due to pre-existing lesions } & \multicolumn{3}{|c|}{ No reported disease progression } \\
\hline & $\begin{array}{l}\text { Eribulin } \\
(n=216)\end{array}$ & $\begin{array}{l}\text { Capecitabine } \\
(n=204)\end{array}$ & $\begin{array}{l}\text { Total } \\
(N=420)\end{array}$ & $\begin{array}{l}\text { Eribulin } \\
(n=131)\end{array}$ & $\begin{array}{l}\text { Capecitabine } \\
(n=112)\end{array}$ & $\begin{array}{l}\text { Total } \\
(N=243)\end{array}$ & $\begin{array}{l}\text { Eribulin } \\
(n=207)\end{array}$ & $\begin{array}{l}\text { Capecitabine } \\
(n=232)\end{array}$ & $\begin{array}{l}\text { Total } \\
(N=439)\end{array}$ \\
\hline $\begin{array}{l}\text { Median OS, months } \\
(95 \% \mathrm{Cl})\end{array}$ & $\begin{array}{l}14.6 \\
(12.4,17.1)\end{array}$ & $\begin{array}{l}11.3 \\
(9.2,13.4)\end{array}$ & $\begin{array}{l}13.0 \\
(11.6,14.4)\end{array}$ & $\begin{array}{l}18.0 \\
(14.7,19.7)\end{array}$ & $\begin{array}{l}16.7 \\
(14.2,19.9)\end{array}$ & $\begin{array}{l}17.1 \\
(15.5,19.4)\end{array}$ & $\begin{array}{l}17.6 \\
(15.4,20.7)\end{array}$ & $\begin{array}{l}16.8 \\
(14.3,19.1)\end{array}$ & $\begin{array}{l}16.9 \\
(15.7,18.9)\end{array}$ \\
\hline $\begin{array}{l}\text { HR eribulin vs. capecitabine } \\
(95 \% \mathrm{Cl})\end{array}$ & $\begin{array}{l}0.75 \\
(0.61,0.93)\end{array}$ & & - & $\begin{array}{l}0.92 \\
(0.69,1.23)\end{array}$ & & - & $\begin{array}{l}0.91 \\
(0.73,1.15)\end{array}$ & & - \\
\hline$p$ value & 0.008 & & - & 0.57 & & - & 0.44 & & - \\
\hline
\end{tabular}

Progression events were determined by independent review

$\mathrm{Cl}$ confidence interval, $H R$ hazard ratio, OS overall survival 


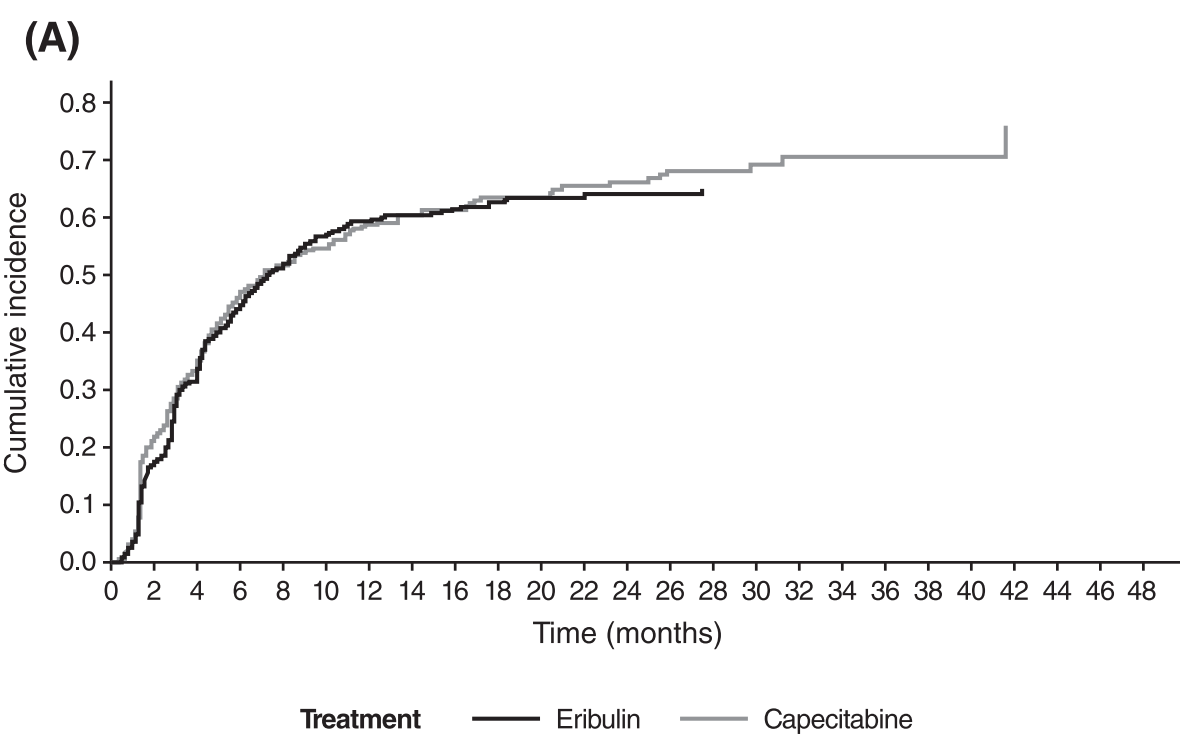

(B)

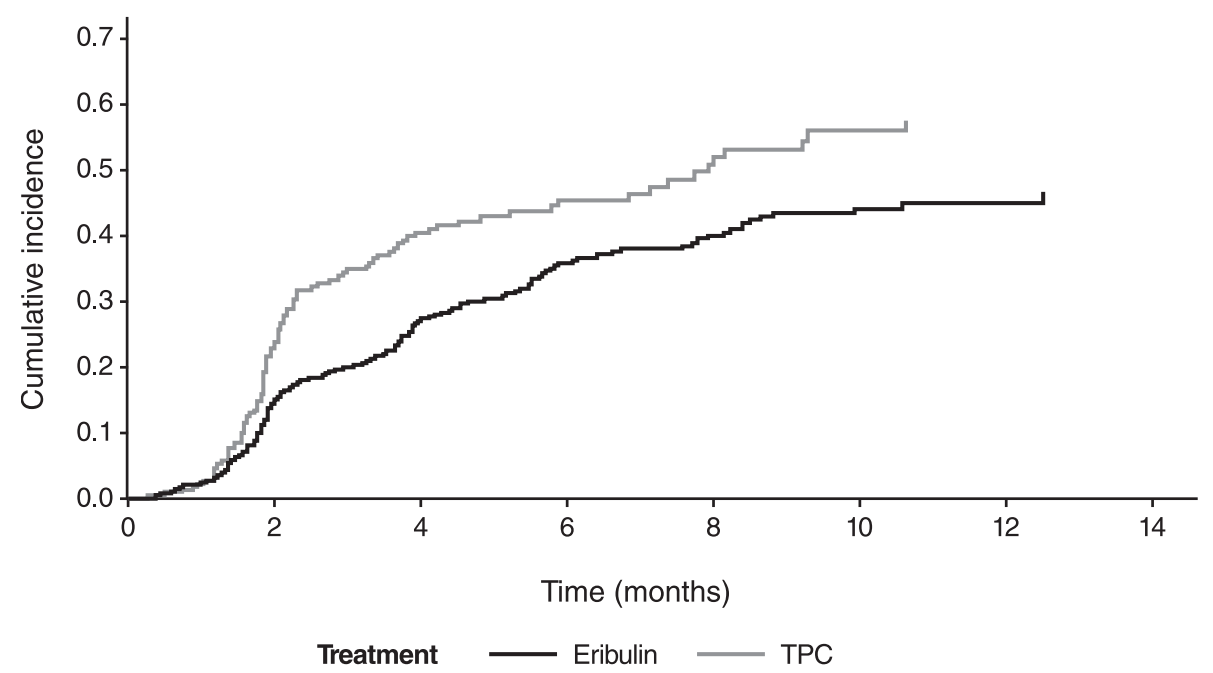

EMBRACE; Eisai Metastatic Breast Cancer Study Assessing Physician’s Choice Versus E7389;

TPC, treatment of physician's choice.

Fig. 1 Cumulative incidence function of new metastasis or death in studies (a) 301, and (b) 305/EMBRACE. The data are based on independent review of the intent-to-treat population.

0.0017). Sensitivity analysis of new-metastasis-free survival using log-rank test showed similar results (median 6.4 vs. 4.8 months; HR 0.70; $p=0.006$; Fig. 2b).

There were again some differences in the sites of new metastases between the eribulin and TPC arms (Table 3), but these were modest and not consistent with Study 301, with the exception of patients in the eribulin arm appearing more likely to develop lymph node, breast, and chest wall metastases, and patients in the comparator arm appearing more likely to develop liver and "other" metastases in both Study 305/EMBRACE and Study 301. The time to new metastases in vital organs favored eribulin over TPC (HR 0.63; $p=0.04$; Fig. 3b).

\section{Comparison of eribulin versus TPC according to treatment duration}

The patients whose disease progressed due to the development of a new metastasis were treated with eribulin for a significantly longer duration than those receiving capecitabine (median 139 vs. 68 days; $p<0.001$ ). Similar results were also observed in the subgroups of eribulin and capecitabine patients whose disease progressed due to an 
(A)

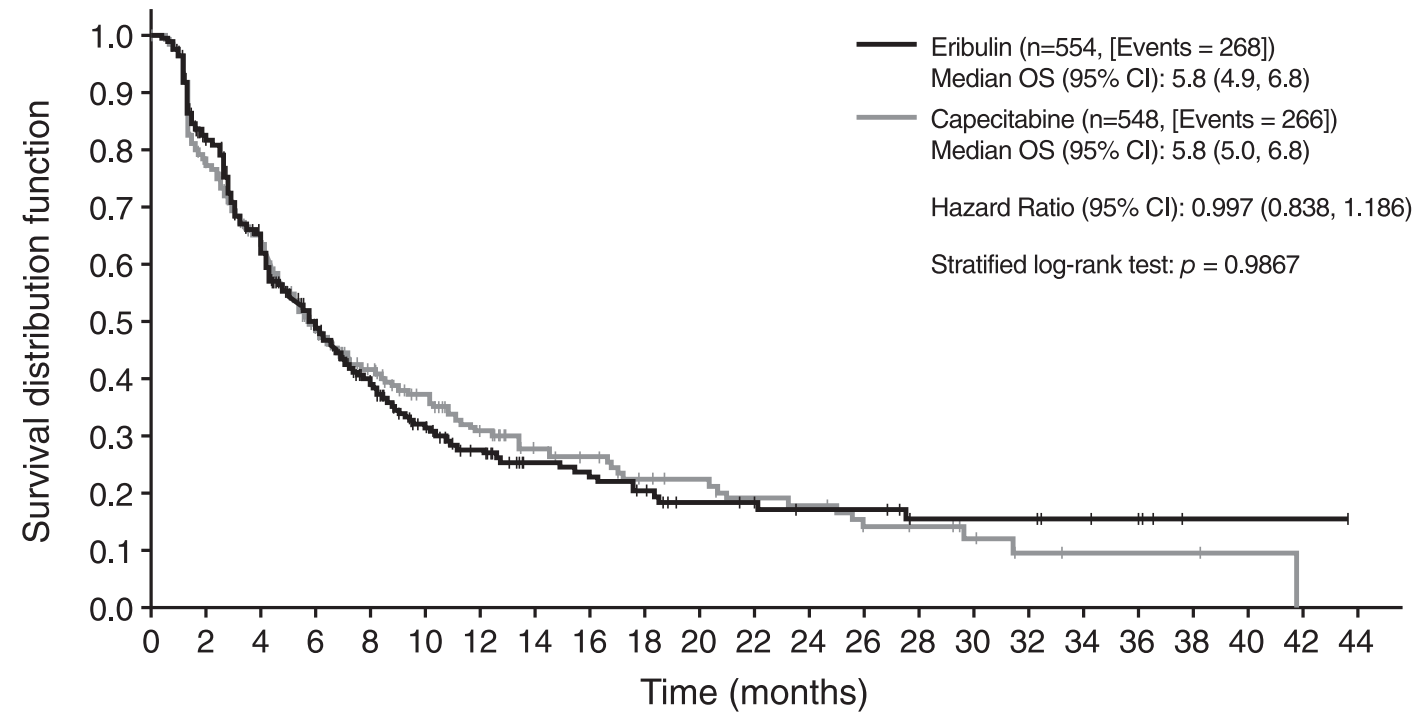

Number of subjects at risk:

$\begin{array}{llllllllllllllllllllllllll}\text { Eribulin } & 554 & 360 & 240 & 136 & 91 & 62 & 45 & 30 & 27 & 23 & 17 & 16 & 12 & 12 & 8 & 8 & 8 & 6 & 5 & 1 & 1 & 1 & 0 \\ \text { Capecitabine } & 548 & 336 & 229 & 126 & 91 & 67 & 50 & 36 & 31 & 22 & 20 & 16 & 15 & 10 & 9 & 6 & 3 & 2 & 2 & 2 & 1 & 0 & 0\end{array}$

(B)

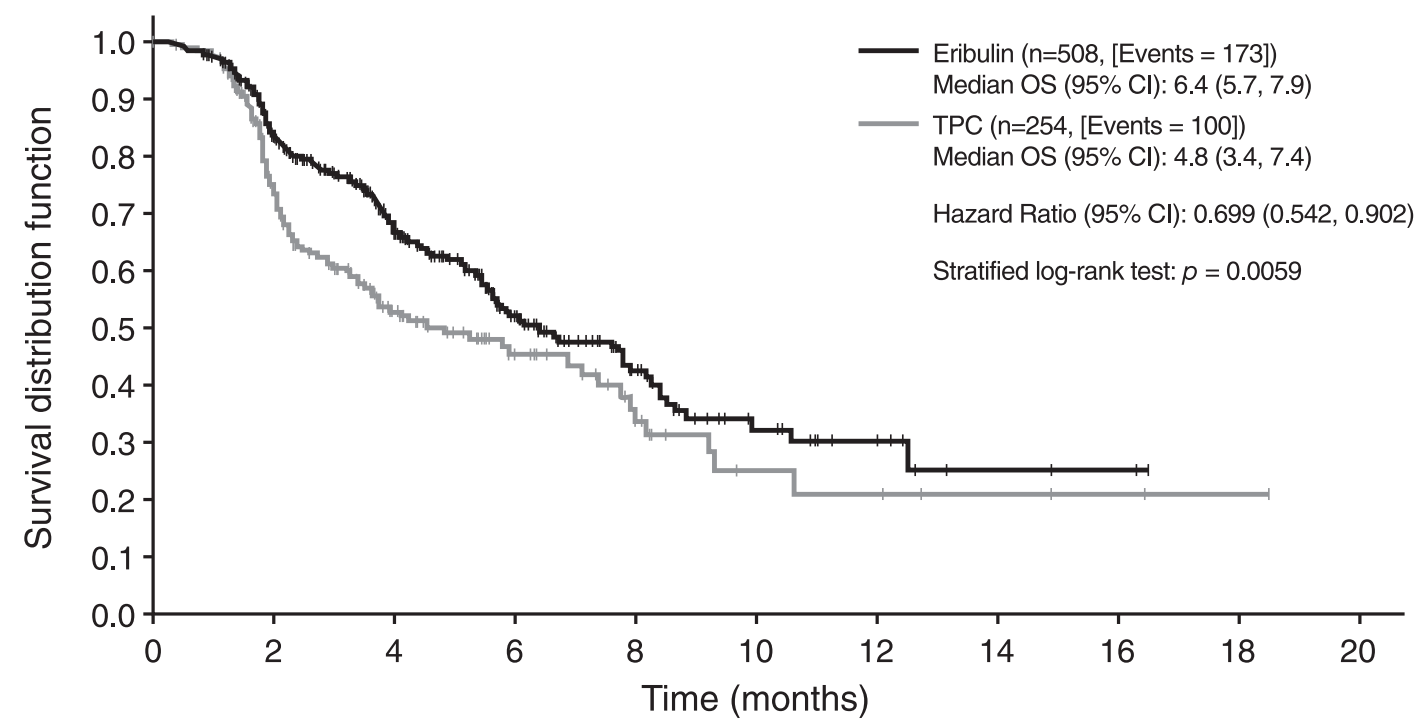

Number of subjects at risk:

$\begin{array}{lrrrrrrrrrr}\text { Eribulin } & 508 & 313 & 174 & 83 & 39 & 18 & 9 & 3 & 2 & 0 \\ \text { TPC } & 254 & 131 & 64 & 33 & 17 & 6 & 5 & 3 & 2 & 1\end{array}$

CI, confidence interval; EMBRACE; Eisai Metastatic Breast Cancer Study Assessing Physician's Choice Versus E7389; OS, overall survival; TPC, treatment of physician's choice.

Fig. 2 New-metasasis-free survival in studies (a) 301 and (b) 305/EMBRACE. The data are based on independent review of the intent-to-treat population. New-metastasis-free survival was defined as the time from randomization to death or disease progression due to a new metastasis (whichever occurred earlier). 
Table 3 Summary of new metastases by site ${ }^{a}$ based on independent review (intent-to-treat populations)

\begin{tabular}{|c|c|c|c|c|}
\hline \multirow[t]{3}{*}{ Parameter } & \multicolumn{2}{|l|}{ Study 301} & \multicolumn{2}{|c|}{ Study 305/EMBRACE } \\
\hline & Eribulin & Capecitabine & Eribulin & TPC \\
\hline & $(n=554)$ & $(n=548)$ & $(n=508)$ & $(n=254)$ \\
\hline Patients whose disease progressed owing to new metastases, $n(\%)$ & $216(39.0 \%)$ & $204(37.2 \%)$ & $139(27.4 \%)$ & $68(26.8 \%)$ \\
\hline \multicolumn{5}{|l|}{ Sites of new metastases ${ }^{\mathrm{b}}, n(\%)$} \\
\hline Liver & $72(33.3)$ & $80(39.2)$ & $41(29.5)$ & $30(44.1)$ \\
\hline Lung & $30(13.9)$ & $33(16.2)$ & $8(5.8)$ & $4(5.9)$ \\
\hline Lymph nodes & $7(3.2)$ & $3(1.5)$ & $16(11.5)$ & $6(8.8)$ \\
\hline Bone & $19(8.8)$ & $16(7.8)$ & $7(5.0)$ & $2(2.9)$ \\
\hline Skin & 66 (30.6) & $62(30.4)$ & $3(2.2)$ & $1(1.5)$ \\
\hline CNS (brain/spine) & $4(1.9)$ & $17(8.3)$ & $7(5.0)$ & 0 \\
\hline Breast & $12(5.6)$ & $7(3.4)$ & $3(2.2)$ & 0 \\
\hline Chest wall & $9(4.2)$ & $4(2.0)$ & $25(18.0)$ & $5(7.4)$ \\
\hline Other & $6(2.8)$ & $13(6.4)$ & $5(3.6)$ & $4(5.9)$ \\
\hline
\end{tabular}

CNS central nervous system, EMBRACE Eisai Metastatic Breast Cancer Study Assessing Physician's Choice Versus E7389, TPC treatment of physician's choice ${ }^{a}$ Only the first new metastasis observed is recorded. If there were multiple new metastases observed at the same time and all determined as earliest, all new metastasis sites were summarized

${ }^{b}$ Percentages for metastasis sites are based on the number of patients in each study arm whose disease progressed owing to new metastases

existing lesion (median: 189 vs. 73 days, respectively; $p<$ 0.01 ) and in those with no reported disease progression (median 174 vs. 107 days, respectively; $p<0.01$ ).

\section{Discussion}

This analysis is, to our knowledge, the first to focus specifically on new metastases as the cause of disease progression and to demonstrate that patients whose disease progresses due to the development of new metastases have worse OS time than those whose disease progresses due to growth of pre-existing metastases and in comparison with patients with no reported disease progression (the latter includes patients who died for reasons other than the development of new metastasis or growth of pre-existing lesions, patients who experienced clinical deterioration and those that were censored). This confirms the clinical impression that patients who develop new metastasis may have a worse prognosis. Current RECIST criteria do not, however, distinguish between the different progression events.

Only a limited number of published clinical studies have described how different progression events affect OS, and their findings have varied. A post hoc analysis of progression events in 246 patients with metastatic renal cell cancer treated with everolimus in the REnal Cell cancer treatment with Oral RAD001 given Daily (RECORD-1) clinical study showed that the development of new lesions and growth of nontarget lesions were both predictive of worse OS $(p<0.001)$ in univariate analyses [30]. In a multivariate analysis, nontarget progression retained statistical significance $(p=0.005)$, but new metastatic lesions had only borderline significance $(p=0.053)$ [30]. In a metastatic colorectal cancer clinical study, the appearance of new lesions or unequivocal progression of nonmeasurable lesions both carried a worse prognosis (regarding survival) than other measures of progression; this analysis was, however, restricted to assessment of the first on-study response assessment [31]. The impact of different time-dependent measures of response on OS was recently described in a series of breast, lung, and colorectal cancer studies [32]. In these analyses, worse OS was significantly associated both with the development of new metastatic lesions $(p<0.001)$ and progression of nontarget lesions $(p<0.001)$.

In contrast, a preliminary report from the phase 3 Intergroup study N9741 in 726 patients with advanced colorectal cancer whose disease progressed on chemotherapy found that individuals whose disease progressed due to the development of new metastases had similar median OS outcome as patients whose disease progressed in preexisting lesions [33].

Our analyses demonstrate that progression due to new metastases is, indeed, associated with significantly worse OS in patients with pretreated MBC. This finding has important potential implications. In the context of clinical studies, it is possible that one type of progression event may be more predictive of OS than other types of progression events. The difference in prognosis, according to the type of progression, might also reflect eligibility criteria: if, for example, the desire is to select patients with a better prognosis, those progressing with new metastases might be excluded. In clinical practice, these data may also help to inform oncologists and their patients when discussing future treatment options and prognosis.

Our second objective was to investigate whether the different types of progression explain the greater impact of eribulin on OS than on PFS. "New-metastasis-free 


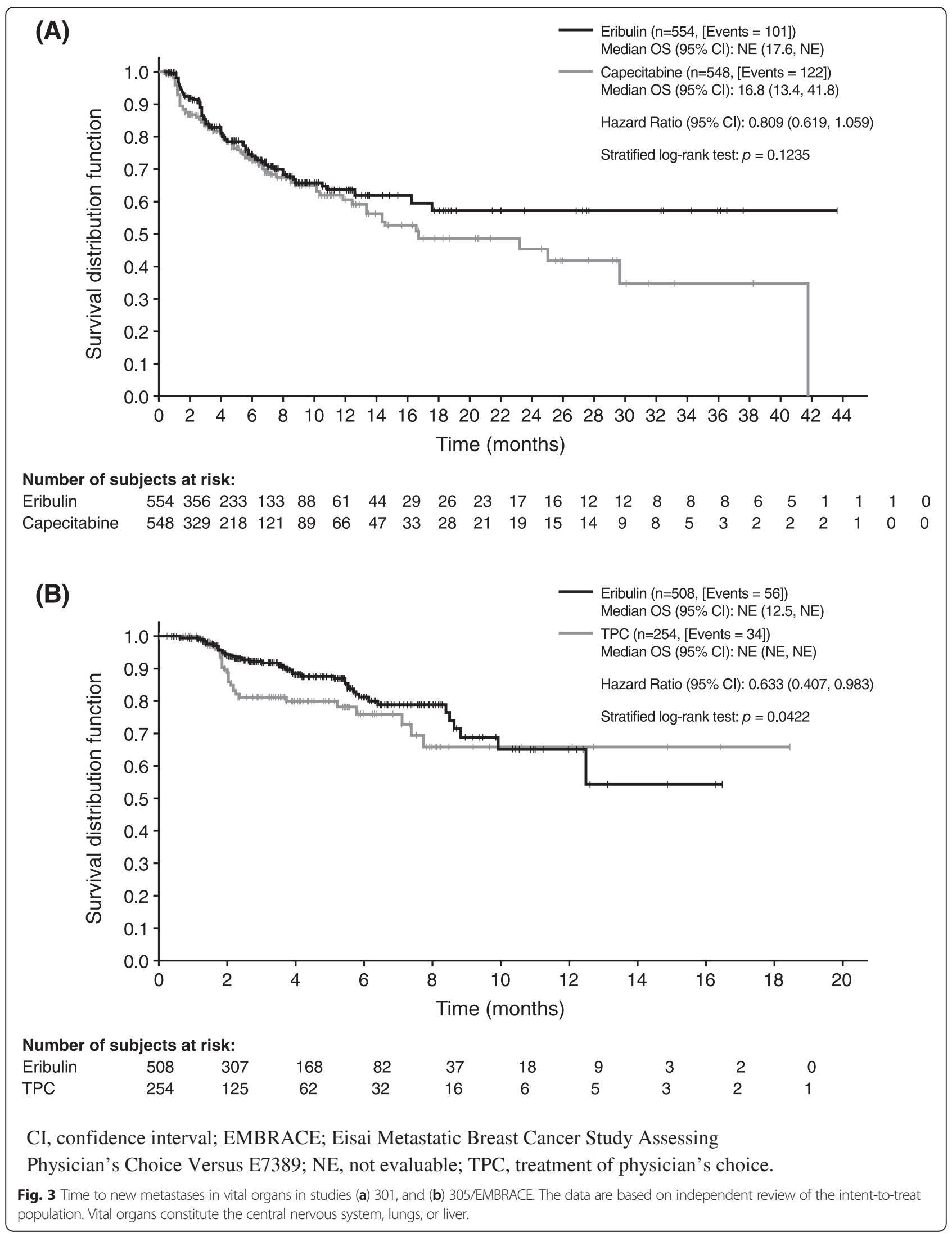


Table 4 Overall survival for different patient groups in Study 305/EMBRACE

\begin{tabular}{|c|c|c|c|c|c|c|c|c|c|}
\hline & \multicolumn{3}{|c|}{ Progression due to new metastases } & \multicolumn{3}{|c|}{ Progression due to pre-existing lesions } & \multicolumn{3}{|c|}{ No reported disease progression } \\
\hline & $\begin{array}{l}\text { Eribulin } \\
(n=139)\end{array}$ & $\begin{array}{l}\text { TPC } \\
(n=68)\end{array}$ & $\begin{array}{l}\text { Total } \\
(N=207)\end{array}$ & $\begin{array}{l}\text { Eribulin } \\
(n=189)\end{array}$ & $\begin{array}{l}\text { TPC } \\
(n=73)\end{array}$ & $\begin{array}{l}\text { Total } \\
(N=262)\end{array}$ & $\begin{array}{l}\text { Eribulin } \\
(n=180)\end{array}$ & $\begin{array}{l}\text { TPC } \\
(n=113)\end{array}$ & $\begin{array}{l}\text { Total } \\
(N=293)\end{array}$ \\
\hline Median OS, months (95\% Cl) & $\begin{array}{l}12.3 \\
(9.9,13.4)\end{array}$ & $\begin{array}{l}8.4 \\
(7.0,14.4)\end{array}$ & $\begin{array}{l}11.1 \\
(8.9,13.1)\end{array}$ & $\begin{array}{l}13.0 \\
(11.1,14.5)\end{array}$ & $\begin{array}{l}11.1 \\
(8.7,15.3)\end{array}$ & $\begin{array}{l}12.2 \\
(11.0,14.2)\end{array}$ & $\begin{array}{l}15.8 \\
(12.1,19.1)\end{array}$ & $\begin{array}{l}10.7 \\
(9.2,14.8)\end{array}$ & $\begin{array}{l}13.0 \\
(11.1,16.9)\end{array}$ \\
\hline HR eribulin vs. TPC (95 \% Cl) & $\begin{array}{l}0.76 \\
(0.51,1.12)\end{array}$ & & - & $\begin{array}{l}0.74 \\
(0.50,1.10)\end{array}$ & & - & $\begin{array}{l}0.77 \\
(0.54,1.10)\end{array}$ & & - \\
\hline$p$ value & 0.16 & & - & 0.14 & & - & 0.16 & & - \\
\hline
\end{tabular}

Progression events were determined by independent review

Cl confidence interval, EMBRACE Eisai Metastatic Breast Cancer Study Assessing Physician's Choice Versus E7389, HR hazard ratio, OS overall survival, TPC treatment of physician's choice

survival," a modified PFS that treats death and new metastases (i.e., the more aggressive type of progression) as the event of interest and progression due to existing lesion as a competing risk, was compared between eribulin and the comparator arms. Using the CICR method, newmetastasis-free survival was longer in the eribulin arm than in the comparator arm for Study 305 but not in Study 301. The log-rank test for comparing hazards of new metastases or death also resulted in a $p$ value of 0.006 in the sensitivity analysis for Study 305. Taken together, our results suggest that the development of new metastases is a risk factor for worsening OS and that the lower incidence rates of new metastasis/death in the eribulin arm may explain, at least in part, the OS benefit associated with eribulin in Study 305. Additionally, in Study 305, patients in the eribulin arm were treated for longer than those in the TPC arm irrespective of the type of progression event, which may also have contributed to better OS.

Although in Study 301 there was no difference in the new-metastasis-free survival between the treatment arms, the different types of progression events may provide some explanation for the OS differences observed between treatment arms. The modest increase in OS with eribulin compared with capecitabine in Study 301 was primarily driven by a significant improvement in OS in the new metastasis subgroup, whereas in Study 305 a similar but nonsignificant increase in OS was observed in the eribulin arm in all three "progression" subgroups. In both clinical studies, the proportion of patients with no reported disease progression was lower in the eribulin arm than in the comparator arm (37.4 \% vs. $42.3 \%$ in Study 301, and $35.4 \%$ vs. $44.5 \%$ in Study 305 ).

Thus, new-metastasis-free survival alone cannot account for the observed discordance between PFS and OS; however, a possible explanation for it may be attributed to the recently identified effects of eribulin on the tumor microenvironment. Recent data in human breast cancer models indicate that eribulin may remodel the tumor microenvironment and vasculature, which may improve its antitumor activity and drug delivery compared with other cytotoxic agents [34]. These changes may also potentially impact on the effects of concomitant and/or subsequent anticancer therapies; further studies may be able to substantiate this hypothesis.

One limitation of our analysis of the impact of the development of new metastases on OS is that it was post hoc rather than preplanned. Further, this analysis was not powered for the subgroups reviewed; subsequently, no definitive conclusions can be drawn. These analyses were, however, prompted by the EMA guidelines. It may also be questioned whether our findings can be generalized to the broader population of patients with $\mathrm{MBC}$. Study 301 and Study 305/EMBRACE were, however, both large well-designed phase 3 studies, with approximately a third (33.6\%) of the patients developing new metastases and more than a quarter $(27.1 \%)$ experiencing progression due to growth of pre-existing lesions. Further, although over half of patients $(57.0 \%)$ were treated with a single cytotoxic agent (i.e., eribulin), the remainder received a range of cytotoxic agents used to treat $\mathrm{MBC}$. Finally, our finding of the association between the development of new metastases and worse OS was consistent across both clinical studies. These retrospective findings warrant further investigation in phase $3 \mathrm{MBC}$ datasets as well as prospective evaluation.

\section{Conclusions}

Patients with $\mathrm{MBC}$ who develop tumor progression with new metastases have a worse prognosis than patients whose disease progresses due to growth of pre-existing lesions and compared with patients with no reported disease progression. This finding has potentially important implications both for the interpretation of clinical study results, especially when PFS is the primary endpoint, and for clinical practice. "New-metastasis-free survival" may be a useful complement to PFS in understanding the dynamic between disease progression and survival. The relationship between the different progression events and OS warrants further investigation.

\section{Abbreviations}

$\mathrm{Cl}$ : confidence interval; CICR: cumulative incidence competing risk; CNS: central nervous system; CONSORT: Consolidated Standards of Reporting 
Trials; CPMP: Committee for Proprietary Medicinal Products; DATECAN: Definition for the Assessment of Time-to-event Endpoints in CANcer trials; DFS: disease-free survival; EMA: European Medicines Agency; EMBRACE: Eisai Metastatic Breast Cancer Study Assessing Physician's Choice Versus E7389; HR: hazard ratio; ICH: International Conference for Harmonisation; ITT: intent-to-treat; MBC: metastatic breast cancer; ORR: objective response rate; OS: overall survival; PFS: progression-free survival; RECIST: Response Evaluation Criteria In Solid Tumors; RECORD-1: REnal Cell cancer treatment with Oral RAD001 given Daily; TPC: treatment of physician's choice; WMA: World Medical Association.

\section{Competing interests}

Christopher Twelves received consultancy fees from AstraZeneca, Eisai Inc., and Pfizer, and honoraria for presenting from Cephalon, Eisai Inc., and Roche. Javier Cortes received consultancy fees from Roche and Celgene, and honoraria from Roche, Celgene, Novartis, and Eisai Inc. Peter A. Kaufman received consultancy fees, research funding and honoraria from Eisai Inc. Louise Yelle received research funding to the Institution from Nektar Therapeutics, Boehringer Ingelheim, Celgene, Roche-Hoffman, Eisai Inc., Genentech, Medlmmune, and Puma Biotechnology Inc. Ahmad Awada participated in advisory boards organized by Eisai Inc. Terri A. Binder is an employee of Eisai Inc. Martin Olivo is an employee of Eisai Inc. James Song is an employee of Eisai Inc. Joyce A. O'Shaughnessy received honorarium from an Eisai Inc. advisory board. Maria Jove has no relevant conflicts of interest to disclose. Edith A. Perez has no relevant conflicts of interest to disclose.

\section{Authors' contributions}

Eisai Inc. funded the studies and supported the authors in the development of the study design, and in the collection, analysis, and interpretation of data. CT, PK, and JC contributed to the study conception and design; CT, EP, JC, PK, LY, and AA contributed to the collection of data. All authors contributed to the analysis and interpretation of data, and the development of the manuscript. Medical writing support was provided by Oxford PharmaGenesis and was supported and funded by Eisai Inc. The authors have had full access to the study data. The decision to submit was taken jointly by the authors and Eisai Inc. All authors read and approved the final manuscript.

\section{Acknowledgements}

We thank Susan McCutcheon, formerly of Eisai Inc., for her contribution in the design of this study, and Yi Philip He, formerly of Eisai Inc., for statistical analysis of the data. In addition, we would like to thank Felix Garzon, formerly of Eisai Inc., for his contributions to the development of the article and input into the study, and Liang Liu of Eisai Inc., for statistical analyses of the data and development of the manuscript.

\section{Disclosure}

Data on patient outcomes from Study 301, reporting on progression with new metastases or increase in size of pre-existing lesions, were presented at the European Cancer Congress 2013 congress in Amsterdam, Netherlands, abstract 1911 .

\section{Author details}

'Section of Oncology and Clinical Research, Leeds Institute of Cancer and Pathology, University of Leeds and Leeds Teaching Hospitals Trust, Leeds, UK. ${ }^{2}$ Department of Oncology, Vall d'Hebron Institute of Oncology, Barcelona, Spain. ${ }^{3}$ Department of Oncology, Ramon y Cajal University Hospital, Madrid, Spain. ${ }^{4}$ Section of Hematology/Oncology, Norris Cotton Cancer Center, Dartmouth-Hitchcock Medical Center, Lebanon, NH, USA. ${ }^{5}$ Department of Medicine, University of Montreal, Montreal, QC, Canada. ${ }^{6}$ Medical Oncology Clinic, Medicine Department, Jules Bordet Institute, Université Libre de Bruxelles, Brussels, Belgium. ${ }^{7}$ Oncology Imaging, Eisai Inc., Woodcliff Lake, NJ, USA. ${ }^{8}$ Oncology PCU, Clinical Development, Eisai Inc., Woodcliff Lake, NJ, USA. ${ }^{9}$ Department of Medical Affairs, Eisai Inc., Woodcliff Lake, NJ, USA. ${ }^{10}$ Department of Medical Oncology, US Oncology, Texas Oncology-Baylor Charles A. Sammons Cancer Center, Dallas, TX, USA. "1Division of Hematology/Oncology, Department of Cancer Biology, Mayo Clinic, Jacksonville, FL, USA. ${ }^{12}$ St James' Institute of Oncology, St James' University Hospital, University of Leeds, Bexley Wing, Level 4, Beckett Street, Leeds, UK.

Received: 24 August 2015 Accepted: 19 November 2015 Published online: 09 December 2015

\section{References}

1. McCain Jr JA. The ongoing evolution of endpoints in oncology. Manag Care. 2010;19(5 Suppl 1):1-11.

2. Ellis LM, Bernstein DS, Voest EE, Berlin JD, Sargent D, Cortazar P, et al. American Society of Clinical Oncology perspective: raising the bar for clinical trials by defining clinically meaningful outcomes. J Clin Oncol. 2014; 32:1277-80.

3. Burzykowski T, Buyse M, Piccart-Gebhart MJ, Sledge G, Carmichael J, Lück $\mathrm{HJ}$, et al. Evaluation of tumor response, disease control, progression-free survival, and time to progression as potential surrogate end points in metastatic breast cancer. J Clin Oncol. 2008;26:1987-92.

4. Broglio KR, Berry DA. Detecting an overall survival benefit that is derived from progression-free survival. J Natl Cancer Inst. 2009;101:1642-9.

5. Palumbo R, Sottotetti F, Riccardi A, Teragni C, Pozzi E, Quaquarini E, et al. Which patients with metastatic breast cancer benefit from subsequent lines of treatment? An update for clinicians. Ther Adv Med Oncol. 2013;5:334-50.

6. Saad ED, Buyse M. Overall survival: patient outcome, therapeutic objective, clinical trial end point, or public health measure? J Clin Oncol. 2012;30: 1750-4.

7. Korn EL, Freidlin B, Abrams JS. Overall survival as the outcome for randomized clinical trials with effective subsequent therapies. J Clin Oncol. 2011;29:2439-42

8. Gourgou-Bourgade S, Cameron D, Poortmans P, Asselain B, Azria D, Cardoso F, et al. Guidelines for time-to-event end point definitions in breast cancer trials: results of the DATECAN initiative (Definition for the Assessment of Time-to-event Endpoints in CANcer trials). Ann Oncol. 2015;26:873-9.

9. Cortazar P, Zhang JJ, Sridhara R, Justice RL, Pazdur R. Relationship between OS and PFS in metastatic breast cancer (MBC): review of FDA submission data. J Clin Oncol. 2011;29(15 Suppl):Abstract 1035.

10. Johnson JR, Ning YM, Farrell A, Justice R, Keegan P, Pazdur R. Accelerated approval of oncology products: the food and drug administration experience. J Natl Cancer Inst. 2011;103:636-44.

11. Booth CM, Eisenhauer EA. Progression-free survival: meaningful or simply measurable? J Clin Oncol. 2012;30:1030-3.

12. Kantoff PW, Higano CS, Shore ND, Berger ER, Small EJ, Penson DF, et al. Sipuleucel-T immunotherapy for castration-resistant prostate cancer. N Engl J Med. 2010;363:411-22.

13. Kantoff PW, Schuetz TJ, Blumenstein BA, Glode LM, Bilhartz DL, Wyand M, et al. Overall survival analysis of a phase $\|$ randomized controlled trial of a Poxviralbased PSA-targeted immunotherapy in metastatic castration-resistant prostate cancer. J Clin Oncol. 2010;28:1099-105.

14. James ND, Caty A, Borre M, Zonnenberg BA, Beuzeboc P, Morris T, et al. Safety and efficacy of the specific endothelin-A receptor antagonist ZD4054 in patients with hormone-resistant prostate cancer and bone metastases who were pain free or mildly symptomatic: a double-blind, placebo-controlled, randomised, phase 2 trial. Eur Urol. 2009;55:1112-23.

15. James ND, Caty A, Payne $H$, Borre M, Zonnenberg BA, Beuzeboc $P$, et al. Final safety and efficacy analysis of the specific endothelin $A$ receptor antagonist zibotentan (ZD4054) in patients with metastatic castrationresistant prostate cancer and bone metastases who were pain-free or mildly symptomatic for pain: a double-blind, placebo-controlled, randomized Phase II trial. BJU Int. 2010;106:966-73.

16. Heinemann V, von Weikersthal LF, Decker T, Kiani A, Vehling-Kaiser U, Al-Batran $S$, et al. Randomized comparison of FOLFIRI plus cetuximab versus FOLFIRI plus bevacizumab as first-line treatment of KRAS wild-type metastatic colorectal cancer: German AlO study KRK-0306 (FIRE-3) [abstract]. J Clin Oncol. 2013; 31(Suppl):Abstr LBA3506.

17. Finley DS, Pouliot F, Pantuck A. Words of wisdom. Re: Overall survival analysis of a phase II randomized controlled trial of a poxviral-based PSA-targeted immunotherapy in metastatic castration-resistant prostate cancer. Kantoff PW, Schuetz TJ, Blumenstein BA, et al. Eur Urol. 2010;58:466.

18. Smith MM, Thompson JE, Castillo M, Cush S, Mukherji SK, Miller $\mathrm{CH}$, et al, MR of recurrent high-grade astrocytomas after intralesional immunotherapy. AJNR Am J Neuroradiol. 1996;17:1065-71.

19. Wolchok JD, Hoos A, O'Day S, Weber JS, Hamid O, Lebbé C, et al. Guidelines for the evaluation of immune therapy activity in solid tumors: immune-related response criteria. Clin Cancer Res. 2009;15:7412-20.

20. Georgoulias V, Androulakis N, Kotsakis A, Hatzidaki D, Syrigos K, Polyzos A, et al. Docetaxel versus docetaxel plus gemcitabine as front-line treatment of patients with advanced non-small cell lung cancer: a randomized, multicenter phase III trial. Lung Cancer. 2008;59:57-63. 
21. Grimison PS, Stockler MR, Thomson DB, Olver IN, Harvey VJ, Gebski VJ, et al. Comparison of two standard chemotherapy regimens for good-prognosis germ cell tumors: updated analysis of a randomized trial. J Natl Cancer Inst. 2010;102:1253-62.

22. Reyno L, Seymour L, Tu D, Dent S, Gelmon K, Walley B, et al. Phase III study of N, N-diethyl-2-[4-(phenylmethyl) phenoxy]ethanamine (BMS-217380-01) combined with doxorubicin versus doxorubicin alone in metastatic/recurrent breast cancer: National Cancer Institute of Canada Clinical Trials Group Study MA.19. J Clin Oncol. 2004;22:269-76.

23. Cortes J, O'Shaughnessy J, Loesch D, Blum JL, Vahdat LT, Petrakova K, et al. Eribulin monotherapy versus treatment of physician's choice in patients with metastatic breast cancer (EMBRACE): a phase 3 open-label randomised study. Lancet. 2011:377:914-23.

24. Kaufman PA, Awada A, Twelves C, Yelle L, Perez EA, Velikova G, et al. Phase III open-label randomized study of eribulin mesylate versus capecitabine in patients with locally advanced or metastatic breast cancer previously treated with an anthracycline and a taxane. J Clin Oncol. 2015;33:594-601.

25. Awada A, Kaufman PA, Yelle L, Cortes J, Wanders J, O'Shaughnessy J, et al. A phase III, open-label, randomized study of eribulin versus capecitabine in patients with metastatic breast cancer: effect of post-progression anti-cancer treatments (PPT) and metastatic progression events on overall survival. San Antonio, TX, USA: Poster presented at the 36th Annual CTRC-AACR San Antonio Breast Cancer Symposium; 2013

26. European Medicines Agency. Appendix 1 to the guidance on the evaluation of anticancer medicinal products in man. 2013. http://www.ema.europa.eu/ docs/en_GB/document_library/Scientific_guideline/2013/01/WC500137126. pdf. Accessed 30 March 2015.

27. Therasse P, Arbuck SG, Eisenhauer EA, Wanders J, Kaplan RS, Rubinstein L, et al. New guidelines to evaluate the response to treatment in solid tumors. European Organization for Research and Treatment of Cancer, National Cancer Institute of the United States, National Cancer Institute of Canada. J Natl Cancer Inst. 2000;92:205-16.

28. Andersen PK, Geskus RB, de Witte T, Putter H. Competing risks in epidemiology: possibilities and pitfalls. Int J Epidemiol. 2012;41:861-70.

29. Gray RJ. A class of K-sample tests for comparing the cumulative incidence of a competing risk. Ann Statist. 1988;16:1141-54.

30. Stein A, Bellmunt J, Escudier B, Kim D, Stergiopoulos SG, Mietlowski W, et al. Survival prediction in everolimus-treated patients with metastatic renal cell carcinoma incorporating tumor burden response in the RECORD-1 trial. Eur Urol. 2013;64:994-1002

31. Suzuki C, Blomqvist L, Sundin A, Jacobsson H, Byström $P$, Berglund A, et al. The initial change in tumor size predicts response and survival in patients with metastatic colorectal cancer treated with combination chemotherapy. Ann Oncol. 2012;23:948-54.

32. Litiere $S$, de Vries EG, Seymour L, Sargent D, Shankar L, Bogaerts J, et al. The components of progression as explanatory variables for overall survival in the Response Evaluation Criteria in Solid Tumours 1.1 database. Eur J Cancer. 2014; 50:1847-53.

33. Grothey A, Heun JM, Branda M, Goldberg RM, Sargent DJ. New lesions versus growth of existing disease: does it impact prognosis? J Clin Oncol. 2011;29(Suppl):Abstr 2579.

34. Funahashi $Y$, Okamoto $K$, Adachi $Y$, Semba T, Uesugi M, Ozawa $Y$, et al. Eribulin mesylate reduces tumor microenvironment abnormality by vascular remodeling in preclinical human breast cancer models. Cancer Sci. 2014; 105:1334-42.

\section{Submit your next manuscript to BioMed Central and we will help you at every step:}

- We accept pre-submission inquiries

- Our selector tool helps you to find the most relevant journal

- We provide round the clock customer support

- Convenient online submission

- Thorough peer review

- Inclusion in PubMed and all major indexing services

- Maximum visibility for your research

Submit your manuscript at www.biomedcentral.com/submit 